\title{
Linear 3 and 5-step methods using Taylor series expansion for solving special 3rd order ODEs
}

\begin{abstract}
Some new linear 3 and 5-step methods for solving special third order ordinary differential equations directly are constructed using Taylor's series expansion. A set of test problems are solved using the new method and the results are compared when the problem is reduced to a system of first order ordinary differential equations and then using the existing Runge-Kutta method. The numerical results have clearly shown the advantage and competency of the new methods.
\end{abstract}

Keyword: Linear 3 and 5-step methods; Taylor series expansion; 3rd order ODEs 